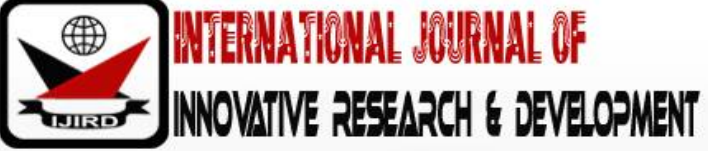

ISSN 2278 - 0211 (Online)

\section{Determination of Cracking Criteria of Palm Nut Based on Impact Mechanics Analysis}

\author{
Amaechi 0. Joseph \\ Lecturer, Department of Industrial Technology, \\ Ignatius Ajuru University of Education, Nigeria
}

\begin{abstract}
:
The cracking unit consists of the region that breaks the kernel by simple impact. It includes a beater arm or cracking impeller and the cracking chamber. Basically, there are two forces that exist on the impeller blade depending on its state of motion. These forces are the centrifugal forces which is concerned with dynamic motion responsible for cracking and static state which is associated with weight of the blade. Some crop physical and mechanical properties are responsible for effective cracking operations. They include: moisture content, size or diameter of nut, thickness of shells, type of nut, nut particle density, cracking rotational speed, feed rate, impeller rim diameter, through put capacity, age of machine etc. Findings shows that relative velocity of normal impact that causes cracking depends on the following criterion: (i) cracking occurs when there is no much rebound of nut and inelastic collision can be assumed (ii) breakage depend on velocity v which dictates the kinetics energy of normal impact (iii) the moisture content decreases, the rate of unbroken kernel increases cracking occurs with the range $r_{1} \leq r \leq r_{2}, r_{2}$. Dura, Tenera and mixture of Dura and Tenera could be cracked at the range of $28 \mathrm{~cm}, 42 \mathrm{~cm}$ and $58 \mathrm{~cm}$ radius on the rotary blade.
\end{abstract}

Keywords: Centrifugal force, impact, velocity, mechanical properties crop physical properties

\section{Introduction}

The centrifugal nut cracker is characterized by increase in kernel breakage, although some of the nuts are discharged without been cracked, Obiakor \& Baba Tunde (1999) Koya (2006). Kernel breakage occurs because the kernel upon release from nut shell rebound in the cracking chamber through the impeller and subjected to secondary impact which induces breakage. In general terms, agricultural products including palm nuts are non-homogenous and some variations occurs in the properties of nut of the same size, therefore, the force required in breaking the nut is not the same, Koya (2006). Similarly, the interactions between adjacent nuts may obstruct the direct impingement of the individual nut to the cracking chamber. Also, the range of impact on the impeller may cause some nut to discharge uncracked. It is experted to obtain high cracking performance if the cracking range on the impeller is determined. Similarly, it is reasonable to expect lower kernel breakage if the nut cracker is driven at low cracking speed, this is to reduce the intensity of the secondary impacts on the kernels which are released after the first impacts are not damaged. Based on this, a cracking process to subject the nut to a repeated but lower impact than the least cracking force is contemplated Faborode (2005). The mechanism of nut cracking in the conventional nut cracker is by multiple impacts from the spontaneous bouncing of the nut. The nut is broken when it is hurled against the cracking chamber by the impeller repeatedly. It is assumed that the nut upon the first impact is deformed beyond the elastic limit to provide the initial crack, Koya (2006). There may be poor state of cracking performance probably because of excessive rotational speed, in the same vain, (Babatunde and Okoli 1988, Jimoh and Olukunle 2013) reports that the hurling speed of 30ms-1 and 26ms-1 were required to crack palm nut of minimum diameter through the impeller blade. The cracking operational devices are identified as roller, hammer impact and centrifuged impact crackers. The roller crackers have two rollers revolving in opposite direction which subjects the palm nut to compressive forces as the nuts moves through the rollers. However, the gaps in the arrangement of the rollers are constant at any preset condition. The hammer impacts cracker breaks or cracks the nut by impact as the hammer falls on the nuts; however, kernel breakage is a major setback in this method of cracking. Various studies have been done on nut impact energy and the related variables that enables cracking to take place effectively.It has also been established that the impact energy required to crack nut in mechanically operated cracker could be evaluated using static impact method. The nut impact energy is given by (Tantis, 2011; Asoegwu, 1995; Dienagha Ibaniehuka, 1991). The study on a functional horizontal shaft cracking machine furtherreveals that cracking taken place when the horizontal shaft makes a force impact on the nut thereby shattering the shell and leaving the kernel.Similarly, Oke (2007) maintain that the cracking unit is made up of feeder hopper, impeller shaft, cracking drum, impeller blade. The nut falls on gravity through the hopper channel into a cracking drum where the cracking process takes place with the aid of impeller blade that flabs the palm nut against the walls of cylindrical cracking drum. The three blades are set at $120^{\circ}$ to each other. 
Subsequently, the cracking operation is characterized by the interaction between the palm nut, Rotating impeller blade and the casing of the chamber. It is assumed that Dura nuts with thick shells are cracked with an impact velocity on the palm nut which hits the casingthrough the blade while tenera samples with thin shells is cracked by the rotating blade as the palm nut travels by gravity to hit the nut in motion. The characteristics of the kernel and impacting surface is determined by the following according to Makajuola 1975, Gazer etal (2002). They maintained.

- As moisture contents decreases the rate of unbroken Kernel increases.

- The type of nut such as Dura or Tenera whose thickness are large or thin is a factor that determines which of the units that does the cracking. It is assured that Tenera is cracked by the blade while Dura sample is cracked by an impact on the cracking case wall.

- Roughness of the rotating shaft is another factor; here cracking is carried in between two rotating helical shafts.

- Impeller shaft diameter does the cracking by an interaction between a stationary and rotating shaft.

- Other characteristics that enables the impeller velocity to perform effectively include; feed rate, age of machine, size of nut, though put capacity, etc.

It is also assumed that some equations provided for different cracking speed based of palm nut based on the nut sizes. Hence some palm nut experience cracks with higher percentage of breakage while some will discharge freely without cracking based on the range of impact on the impeller. The importance is that the palm nut is impacted at a speed $10 \mathrm{w}$ against the cracking chamber, considering that the nut conservative energy is neglected. Therefore, the nut is cracked at the first bound on the impeller ring, Erick et al (2009). Research further shows that the speed of the rotor varies significantly with nut mass hence as the nut mass increases the rotational speed of the rotor reduces. This is to avoid excess Kernel breakage. Subsequently, (Koya 2006, Eric et al 2009) explains that kernel breakage may occur because the Kernelupon release from the nut shell rebound in the cracking chamber is subjected to a secondary impact which induces breakage, to avoid this, the rotational shaft must be kept at a fairly low speed. Similarly, Babatunde Okoli (1988) maintained that the speed for cracking the palm nut depends on the drop height; they further recommended a drop height of $250 \mathrm{~mm}$ at a speed of $29.92 \mathrm{~ms}$.

\section{Materials and Methods}

The development of model for palm nut cracking and separation involves action from the hopper to the impeller blade through to the cracking chamber and discharge through the outlet. It is obvious to determine the minimum height to drop a weight or the minimum speed to hurl the palm nut at once. A lower drop height and rotational speed may be required where by impact causes crack nucleation while subsequent impacts accounts for accounts propagation and eventual fracture of the nut shell. This procedure ensures that nut cracking takes place at a minimum impact load, and reduces kernel breakage.It is assumed that the nut is impacted through the impeller blade by a load which is dropped from a certain height and without bouncing or contacting the palm nut. The magnitude of the weight is above the maximum force that will not crack the nut irrespective of the falling height. At the initiation of crack, assuming no energy is lost during the impact, the kinetic energy of the falling weight is changed into elastic strain energy of the nut therefore; Koya (2006) reported that $\mathrm{w}(\mathrm{h}-\mathrm{d} \mathrm{t} \max )=1 / 2\left(\int \max \right) \int \max ----(1)$ where $\mathrm{w}$ in $\mathrm{N}=$ the falling weight, dinm $=$ the diameter of the nut, $\mathrm{h}$ in $\mathrm{m}=$ the falling height $\int \mathrm{max}=$ maximum deformation of nut when gradually compressed by a load $\mathrm{w}$ in $\mathrm{N}$ and $\mathrm{S}$ in $\mathrm{NM}^{-1}$ becomes the stiffness of the material. The impact mechanisms analysis and velocity graphs considered the angle and plane of rotation and imp-act by the impeller blade at a given range (Amaechi, 2019). The velocity of the falling palm nut with respect to time and position is a major consideration in the determination of the range at which cracking occurs. Henceangular velocity is denoted by wwhereas linear velocity is denoted by v. They are graphically represented thus:

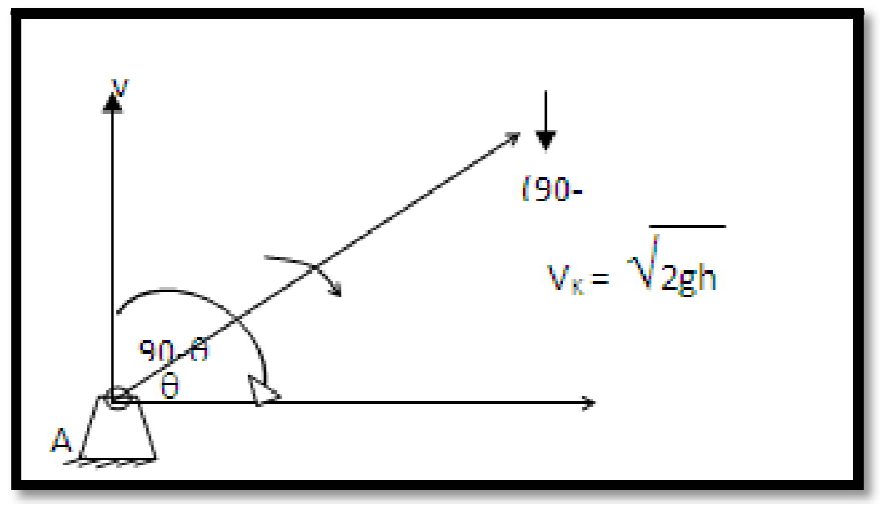

Figure 1: A Link in Pure Rotation of a Plane 


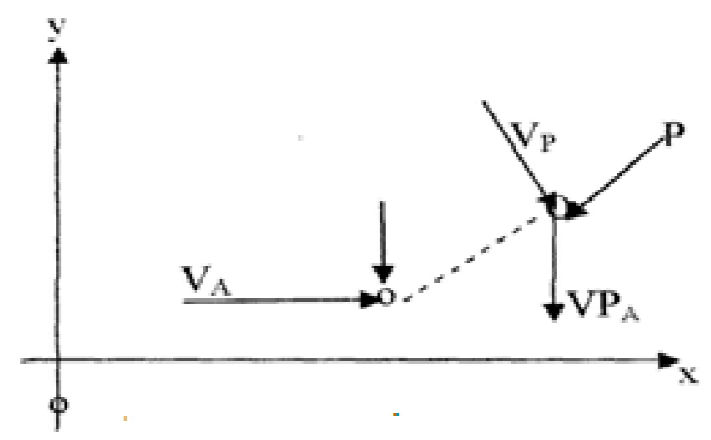

Figure 2: Relative Velocity

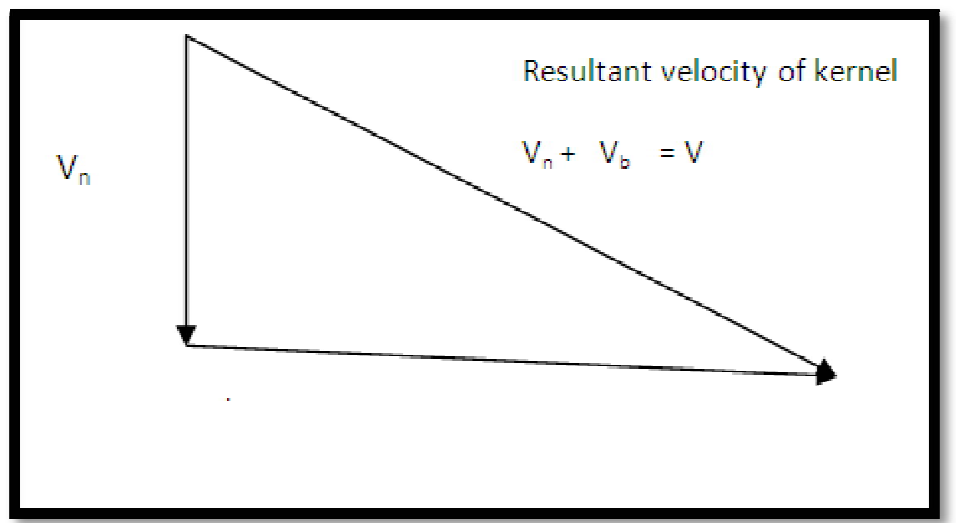

Figure 3: Graphic Velocities of the Blade \& Kernel

2.1. Resultant velocity of Kernel

$$
\begin{aligned}
& \mathrm{V}=\mathrm{V}_{\mathrm{n}}=\mathrm{V}_{\mathrm{b}} \\
& \mathrm{V}_{\mathrm{n}}=\sqrt{2 g h} \\
& \mathrm{~V}_{\mathrm{b}}=\omega r \sqrt{2 g h} \\
& \mathrm{~V}>\mathrm{V}_{\mathrm{b}} \\
& \mathrm{V}>\mathrm{Vn} \\
& \}
\end{aligned}
$$

2.2. Impact Mechanics of Palm Nut

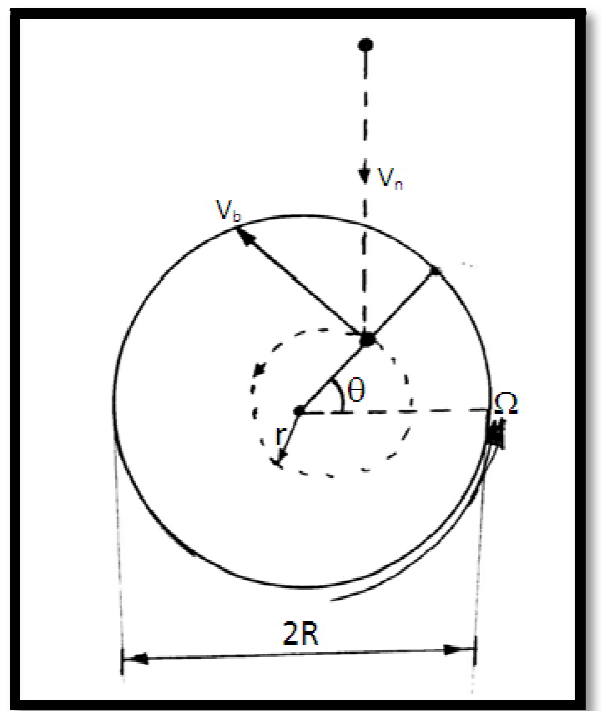

Figure 4: Rotational Velocity of Blade and Nut in the Cracking Chamber

The nut falls vertically with velocity $\mathrm{V}_{\mathrm{n}}$ and strikes the rotating blade. The tangential velocity of the blade at radial location $\mathrm{r}$ is designated $\mathrm{V}_{\mathrm{b}}$. 
From equation (2) above,

$\mathrm{V}=\mathrm{V}_{\mathrm{n}}=\mathrm{V}_{\mathrm{b}}$.

The tangential velocity $\mathrm{V}_{\mathrm{b}}$ becomes $\mathrm{V}=\mathrm{V}_{\mathrm{b}}\left(-\mathrm{V}_{\mathrm{n}} \operatorname{Cos} \theta\right)=\mathrm{Vb}_{+} \mathrm{V}_{\mathrm{n}} \operatorname{Cos} \theta$

$\mathrm{V}_{\mathrm{b}}=\omega \mathrm{r}=\frac{\pi \Omega r}{30}$

It is seen from the system geometry that velocity of free fall of impact

$\mathrm{V}_{\mathrm{n}}=\sqrt{2 g h}=\sqrt{2 g(H-r \operatorname{Sin} \theta)}$

The relative velocity of normal impact is as shown in equation 1 above substituting equation(3.14) (3.15) and(3.16) gives $\mathrm{V}=\frac{\pi \Omega r}{30}+\sqrt{2 g(H-r \operatorname{Sin} \theta)} \cos \theta-$

If there is cracking, there will not be much rebound of nut and inelastic collision can be assumed. Therefore, breakage depends on velocity $\mathrm{v}$ which in turn dictates the kinetic energy of normal impact. If the breakage energy is $E_{b}$, then the threshold velocity for breakage is

$\mathrm{V}=\sqrt{\frac{2 E_{b}}{m}}$

Combing equations (3.17) and(3.18) above we have

$\sqrt{\frac{2 E_{b}}{m}}=\frac{\pi \Omega r}{30}+\sqrt{2 g(H-r \operatorname{Sin} \theta)} \cos \theta \quad-$

To cover for the case where the blade is expected to lie in any of the four quadrants, equation (11) becomes

$\frac{\pi \Omega r}{30}[\operatorname{Sin}(\theta+90)]+(\sqrt{2 g(H-r \operatorname{Sin} \theta)}) \operatorname{Cos} \theta-\sqrt{\frac{2 E_{b}}{m}}=0$

Which gives

$\frac{\pi \Omega r}{30} \operatorname{Sgn}(\operatorname{Cos} \theta)+\operatorname{Sgn}(\operatorname{Cos} \theta) \sqrt{2 g(H-r \operatorname{Sin} \theta)} \operatorname{Cos} \theta-\sqrt{\frac{2 E_{b}}{m}}-$

Applying Taylor series to the second term, equation (3.20) becomes

$\frac{\pi \Omega r}{30}(\operatorname{Cos} \theta)+(\sqrt{2 g H}) \operatorname{Cos} \theta-\frac{g \operatorname{Sin} \theta \operatorname{Cos} \theta r}{\sqrt{2 g H}}-\frac{g^{2} \operatorname{Sin}^{2} \theta \operatorname{Cos} r^{2}}{(2 g H)^{3 / 2}}-\sqrt{\frac{2 E_{b}}{m}}=0$

Where as $\Omega=$ is rotational speed in $[\mathrm{rpm}]$

$\mathrm{V}_{\mathrm{n}}$ is the velocity of free fall the nut in $\left[\mathrm{ms}^{-1}\right]$

$\mathrm{V}_{\mathrm{b}}$ is the tangential velocity of the blade $\left[\mathrm{ms}^{-1}\right]$

$r$ is a general radial location in $(\mathrm{m})$

$\mathrm{R}$ is the radius of the rotor speed $(\mathrm{m})$

$\theta$ is instantaneous angular displacement [rads]

$\mathrm{m}$ is the mass of nut $(\mathrm{kg})$

$\mathrm{g}$ is the acceleration of free fall $\left[\mathrm{ms}^{-1}\right]$

$\mathrm{H}$ is the nut release height $(\mathrm{m}]$

$\mathrm{E}_{\mathrm{b}}$ is the breakage energy $(\mathrm{J})$

$\mathrm{f}(\mathrm{r})$ is the cracking criteria

This is seen to be a quadratic equation in $r$.

If the (LHS) of the equation is represented as

$\mathrm{F}(\mathrm{r})$, then cracking occurs if $\mathrm{F}(\mathrm{r}) \geq 0 \quad$ -

The question becomes; given rotational speed $\Omega$ and known breakage energy $E_{b}$, what is the minimum $r$ and range of angles $\theta$ that will guarantee cracking.

Consider the special cases of equations (12) and (13) above

When $\theta=0, \pi / 2, \pi$ and $3 \pi / 2$;

When $\theta=0$, then $\frac{\pi \Omega r}{30}+\sqrt{2 g H}-\sqrt{\frac{2 E_{b}}{m}} \geq 0$.

$\mathrm{r} \geq \frac{30}{\pi \Omega}+\left(\sqrt{\frac{2 E_{b}}{m}}-\sqrt{2 g H}\right) \quad-\quad \ldots \quad-\quad \ldots$ 
Thus, cracking is possible at some value of $\mathrm{r}$ in the range $0<\mathrm{r}<\mathrm{R}$ as long as $\sqrt{\frac{2 E_{b}}{m}}-\sqrt{2 g H}>0$; which is a realistic condition given the ranges of values of $\mathrm{m}, \mathrm{E}_{\mathrm{b}}$ and $\mathrm{H}$.

When $\theta=\pi$ then $\frac{\pi \Omega r}{30} \sqrt{2 g H}-\sqrt{\frac{2 E_{b}}{m}} \geq 0$.

$\mathrm{r} \leq \frac{30}{\pi \Omega}\left(-\sqrt{\frac{2 g H}{m}}-\sqrt{\frac{2 E_{b}}{m}}\right)$

This is not feasible as it is certain that $\left(-2 g H-\sqrt{\frac{2 E_{b}}{m}}\right)<0$

Based on the above, cracking could happen when $0<\theta<\pi / 2$ and $3 \pi / 2 \quad \varangle \theta<2 \pi$.

Theories of the analysis of $F(r)$ i.e. cracking values $F(r)$ is quadratic function which should have positive value for cracking to occur. The solution to the equation $\mathrm{F}(\mathrm{r})=0$ are $\mathrm{r}_{1}$ and $\mathrm{r}_{2}$.

Therefore, plot of $F(r)$ against $r$ will cross the axis at points $r_{1}$ and $r_{2}$. Since the $r$ axis $F(r)$ is a negative quadratic function, Cracking is expected to occur when $r$ is between $r_{1}$ and $r_{2}$. Thus, it can be stated that cracking occurs when the range $r_{1} \leq r \leq$ $r_{2}, r_{2}$ is expected to be very large because of relatively small release height and will not be normally visible in the plot of cracking criteria $\mathrm{F}(\mathrm{r})$.If this condition is not satisfied then the chosen value of $\theta$ is not in the favourable range or $\Omega$ is too small to cause cracking

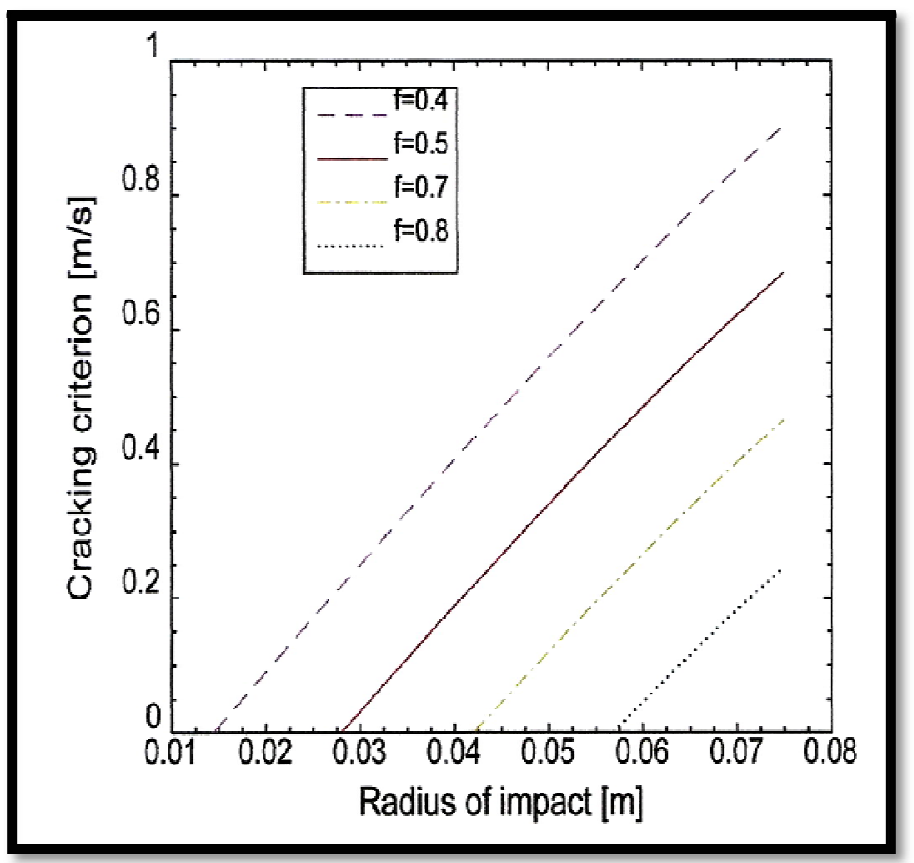

Figure 5

The graph reveals that the cracking impact is initiated within the ranges of $r-15,28,42$ and $58 \mathrm{~cm}$ for cracking criterion of $\mathrm{F}=0.4,0.5,0.7$ and 0.8 respectively.

This further shows that the Tenera palm nut could be cracked within the range of $15 \mathrm{~cm}$ radius on the rotary blade. Again, mixture of Tenera and Dura palm nut could be cracked within the range of $28 \mathrm{~cm}$ radius on the rotary blade while Dura palm nut could be cracked within the range of 42 and $58 \mathrm{~cm}$ radius on the rotary blade respectively. Similarly, it was discovered that as palm nut falls freely from the hopper and strikes the rotary blade tangentially at a radial location $\mathrm{r}$, cracking occurs within the range of the cracking blade when $r 1 \leq r \leq r 20<0 \pi / 2,3 \pi / 2<\theta 2$ $\pi$.

\section{References}

i. Amaechi, O.J. (2019) Performance Modelling of Horizontal-Shaft rotten palm kernel cracking machine unpublished (Ph.D. thesis). Department of Mechanical Engineering, University of Nigeria Nsukka.

ii. Asoegwu, S.N. (1995). Physical properties and cracking energy of energy of conophor nuts at different moisture contents.International agrophysics 9, 131-142.

iii. Babatunde, O.O. and J.O. Okoli (1988). Investigation into effect of nut size on the speed needed for cracking palm nut centrifugal nut cracker. 
iv. Dienagha, A.R.S. and Ibanichuka, O.O. (1991). Energy requirement for palm nut cracking.Journal of the Nigerian Engineer.Vol. 26 (3) 39-46.

v. Erick, F., Simions A, Elias K.S., (2009). The Determination of some design parameter for palm nut crackers. European Journal of Scientific Research. Vol. 38 (2).

vi. Gezer, T.H. Halyseferogullary, and F. Demos some physical properties of Hacychaliloglu apricot pit and its kernel. Journal of food Engineering56. 49-57.

vii. Jimoh, M.O. and O.J. Oku Olakunle (2013). Design of an effective automated machine for quality palm kernel production. IQSR, Journal of mechanical and Civil Engineering (IQSR-JMCE) Vol. 6 pp 88-97.

viii. Koya, O.A. (2006). Palm nut cracking under repeated impact load. Journal of Applied Sciences. 6 (11), 2471-2475.

ix. Makanjola, G.A. (1975). An Evaluation of some centrifugal impaction devices for shelling melon seeds. Journal of Agricultural Engineering Research, 20 (1), 71-72.

x. Obiakor, S.I. and Babatunde, O.O. (1999), Development and testing of the NCAM centrifugal palm nut cracker. AGRIMECH Research and information bulletin of the National centre for Agricultural mechanization (NCAM).

xi. Oke, P.K. (2007). Development and performance evaluation of indigenous palm kernel Dura processing machine. Journal of Engineering and applied Sciences 2 (4): 701-708. 